\title{
Channeling, volume reflection, and volume capture study of electrons in a bent silicon crystal
}

\author{
T. N. Wistisen and U. I. Uggerhøj \\ Department of Physics and Astronomy, Aarhus University, 8000 Aarhus, Denmark \\ U. Wienands," T. W. Markiewicz, R. J. Noble, B. C. Benson, and T. Smith \\ SLAC National Accelerator Laboratory, 2575 Sand Hill Road, Menlo Park, California 94025, USA \\ E. Bagli, L. Bandiera, G. Germogli, V. Guidi, and A. Mazzolari \\ Department of Physics and Earth Sciences of the University of Ferrara, and INFN Section of Ferrara, \\ Via Saragat 1/C, I-44122 Ferrara, Italy \\ R. Holtzapple and S. Tucker \\ California Polytechnic State University, San Luis Obispo, California 93407, USA
}

(Received 28 September 2015; published 5 July 2016)

\begin{abstract}
We present the experimental data and analysis of experiments conducted at SLAC National Accelerator Laboratory investigating the processes of channeling, volume-reflection and volume-capture along the (111) plane in a strongly bent quasimosaic silicon crystal. These phenomena were investigated at 5 energies: $3.35,4.2,6.3,10.5$, and $14.0 \mathrm{GeV}$ with a crystal with bending radius of $0.15 \mathrm{~m}$, corresponding to curvatures of $0.053,0.066,0.099,0.16$, and 0.22 times the critical curvature, respectively. Based on the parameters of fitting functions we have extracted important parameters describing the channeling process such as the dechanneling length, the angle of volume reflection, the surface transmission, and the widths of the distribution of channeled particles parallel and orthogonal to the plane.
\end{abstract}

DOI: 10.1103/PhysRevAccelBeams.19.071001

\section{INTRODUCTION}

Channeling in bent crystals has been thoroughly studied for protons with the purpose of, e.g., proton extraction at accelerator facilities [1-3] and for collimation [4-6]. Much less is known about the efficiency of channeling of electrons in bent crystals. In this paper we present a quantitative investigation of channeling and related phenomena in a strongly bent silicon crystal. The ordered structure of the crystal lattice gives unique access to electromagnetic field strengths otherwise experimentally inaccessible [7] which can be manipulated by e.g. bending of the crystal, as in this experiment, or otherwise inducing a strain in the crystal as exploited in certain new types of crystalline undulators [8-13]. Understanding the dynamics of the electron motion in a bent crystal is important both for the application of bent crystals, but also in order to obtain a better understanding of the dynamics in e.g. crystalline undulators. This paper is a continuation of [14]. Here we include experiments at three

\footnotetext{
*Present address: Argonne National Laboratory, 9700 S. Cass Avenue, Argonne, IL 60439, USA.

Published by the American Physical Society under the terms of the Creative Commons Attribution 3.0 License. Further distribution of this work must maintain attribution to the author(s) and the published article's title, journal citation, and DOI.
}

other energies conducted after the submission of [14], thus presenting a far more detailed study.

\section{THEORY}

Channeling is a phenomenon which occurs in aligned single crystals. If an impinging particle is traveling along a direction of symmetry in the crystal, the motion will no longer be governed by the particle's interaction with an individual atom, but the coherent interaction of many atoms along a crystalline axis or plane. This allows one to average the potential of each atom over the general direction of motion (along the axis or plane), yielding the "continuum" transverse potential. An often employed model of the planar (111) continuum potential is the thermally averaged Doyle-Turner potential of the form [15]

$$
U(x)=2 \sqrt{\pi} Z_{1} \frac{e^{2}}{a_{0}} a_{0}^{2} N d_{p} \sum_{i=1}^{4} \frac{a_{i}}{\sqrt{B_{i}+\rho^{2}}} e^{-\frac{x^{2}}{B_{i}+\rho^{2}}},
$$

where $Z_{1}$ is the charge number of the projectile, $\rho=$ $0.062 \AA$ is the r.m.s. thermal vibrational amplitude at room temperature for $\mathrm{Si}, a_{0}=0.53 \AA$ is the Bohr radius, $N$ is the number density of atoms, and $B_{i}=b_{i} / 4 \pi^{2}$ and $x$ is the coordinate transverse to the plane. See Table I for the values of $a_{i}$ and $b_{i}$. A plot of this potential versus transverse position is shown in Figure 1. For Si (111) there are two 
TABLE I. A table of parameters for the Doyle-Turner potential for the Si potential of Eq. (1).

\begin{tabular}{lcccc}
\hline \hline$i$ & 1 & 2 & 3 & 4 \\
\hline$a_{i}[\AA]$ & 2.1293 & 2.533 & 0.8349 & 0.3216 \\
$b_{i}\left[\AA^{2}\right]$ & 57.7748 & 16.4756 & 2.8796 & 0.3860 \\
\hline \hline
\end{tabular}

planar distances given by $d_{1}=\frac{a}{4 \sqrt{3}}$ and $d_{2}=\frac{a \sqrt{3}}{4}$ with $a=5.43 \AA$ being the lattice constant for $\mathrm{Si}$ and $d_{p}=\frac{d_{1}+d_{2}}{2}$. For Si the coefficients are given by [15].

The potential Eq. (1) depends only on one coordinate, transverse to the (111) plane. We define the "transverse mechanical energy" as

$$
E_{\perp}=\frac{p_{\perp}^{2}}{2 \gamma m}+U(x)
$$

which is a conserved quantity to leading order in $\frac{1}{\gamma^{2}}$. Here $p_{\perp}$ is the momentum component normal to the plane and $\gamma$ is the relativistic Lorentz factor of the impinging electron. The critical angle is defined as the largest angle the electron can have with the plane while still being bound in the transverse potential. From Eq. (2) one obtains

$$
\theta_{c}=\frac{p_{\perp, \max }}{p}=\sqrt{\frac{2 U_{0}}{E \beta^{2}}},
$$

where $U_{0}$ is the depth of the potential, $E$ the energy of the impinging electron and $\beta=\frac{v}{c}$ its velocity in units of $c$, the speed of light. In a bent crystal, channeling is easily described in a rotating coordinate system such that the $x$-coordinate is the displacement from the bent channel in the radial direction of the bending arc. This choice of coordinate system gives rise to a centrifugal force. For a relativistic particle this is equivalent to a centrifugal barrier potential of the form

$$
U_{\mathrm{CF}}(x)=\frac{E x}{R},
$$

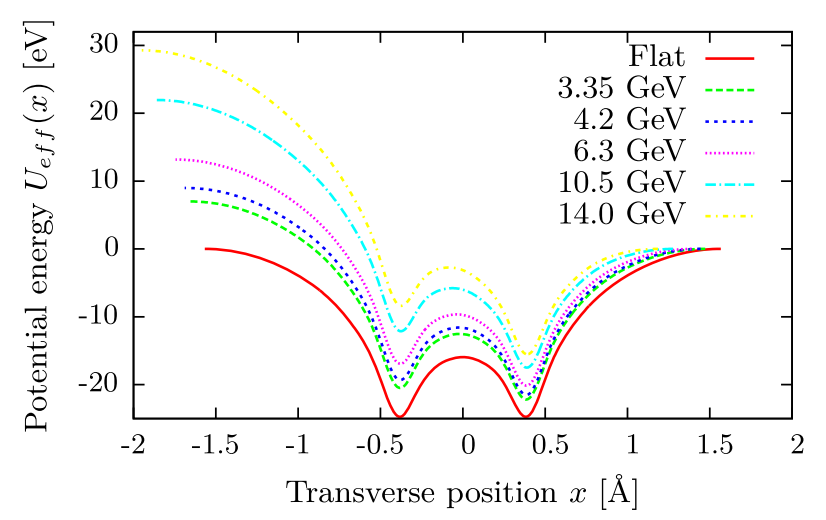

FIG. 1. A plot of the effective potential of Eq. (5) for a flat crystal and for the relevant electron energies with bending radius $R=0.15 \mathrm{~m}$. where $R$ is the bending radius of the crystal. The motion of the channeled particle is therefore described by an effective potential

$$
U_{\mathrm{eff}}(x)=U(x)+\frac{E x}{R}
$$

See Figure 1 for plots of the potential for the relevant energies. It is evident that the bending of the crystal reduces the potential depth and thus the effective critical angle in Eq. (3). If the centrifugal potential barrier of Eq. (4) changes by a significant amount compared to the potential depth of the unbent crystal over an interplanar distance one approaches criticality, and the bending of the crystal cannot be treated as a small perturbation to the unbent case. This is closely related to the critical radius, defined as the radius of curvature where the largest value of the field compensates the centrifugal force. In the case of the (111) plane, there are 2 planar distances, and the critical radius of the largest plane is then given by

$$
R_{c}=\frac{E d_{2}}{4 U_{0}}
$$

Typically the critical radius is calculated based on the more precise Moliere or Doyle-Turner potential see e.g. [16], but in analytical formulas for the dependence of e.g. dechanneling length and surface acceptance on the curvature $R$, the parabolic approximation is used and this is the critical radius in this approximation. From Eq. (6) it is seen that the critical radius for $14.0 \mathrm{GeV}$ is $3.3 \mathrm{~cm}$ which is comparable in magnitude to the $15 \mathrm{~cm}$ radius of curvature of the crystal in this experiment.

\section{A. Dechanneling}

In a crystal of significant length, the simple picture of a conserved transverse mechanical energy is too simple. Multiple scattering of the channeled particle leads to an increasing transverse mechanical energy that results in the particle leaving the channel and the motion is no longer bound. The typical length over which this happens is called the dechanneling length and its size is found by equating the scattering angle due to multiple scattering to the critical angle of Eq. (3). The result is given by [17]

$$
L_{d}=\frac{\alpha U_{0} E}{\pi\left(m c^{2}\right)^{2}} X_{0}
$$

where $\alpha$ is the fine structure constant and $X_{0}$ the radiation length in the amorphous medium. A simple model of dechanneling is obtained by assuming a constant probability per unit length per particle to dechannel, $\frac{1}{L_{d}}$, giving an exponential decay of the number of particles in the channel $[18,19]$, 


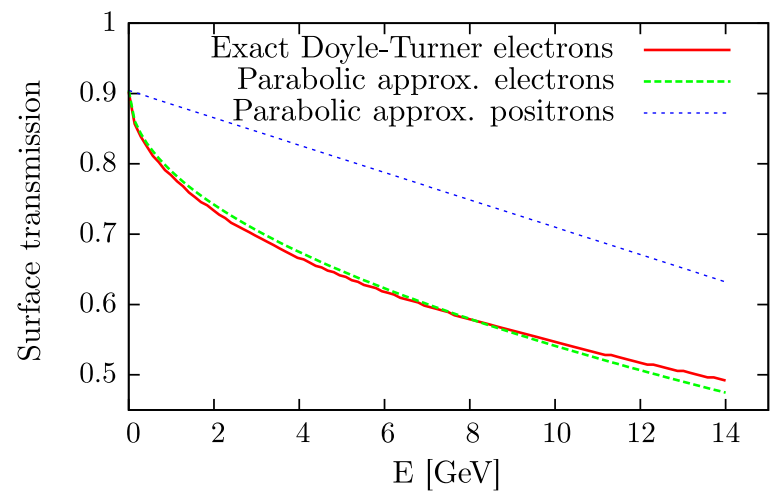

FIG. 2. A figure comparing the surface transmission for electrons using the exact Doyle-Turner potential and the parabolic approximation. We have also plotted the parabolic approximation for positrons to show the difference between positive and negative particles.

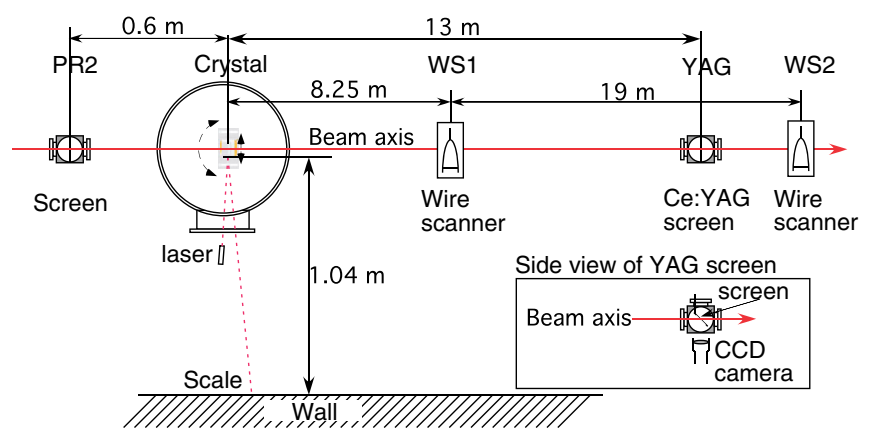

FIG. 3. A sketch of the experimental setup.

$$
N(z)=N_{0} e^{-\frac{z}{L_{d}}}
$$

Equation (8) is for the dechanneling length in a straight crystal. This simple model can easily be modified to the case of a bent crystal. From Eq. (5) it is seen that the potential depth from the case of a straight crystal is, to first order in $\frac{1}{R}$, reduced by $\frac{E d_{2}}{2 R}=\frac{2 R_{\text {crit }}}{R} U_{0}$ such that the dechanneling length is instead given by

$$
L_{d, b}=\frac{\alpha U_{0} E}{\pi m^{2}} X_{0}\left(1-\frac{2 R_{\mathrm{crit}}}{R}\right),
$$

which is applicable while $R \gg 2 R_{\text {crit }}$. By comparing this formula obtained by using the parabolic approximation to the exact result obtained numerically using the DoyleTurner potential, there is a discrepancy of at most $13 \%$ at the highest energy.

\section{B. Volume capture and volume reflection}

The phenomenon of volume capture in a bent crystal is the process where a particle, initially unbound, scatters and loses transverse mechanical energy to become bound - "captured" in the channel. As such it is closely related to the dechanneling phenomenon [20,21]. Volume capture

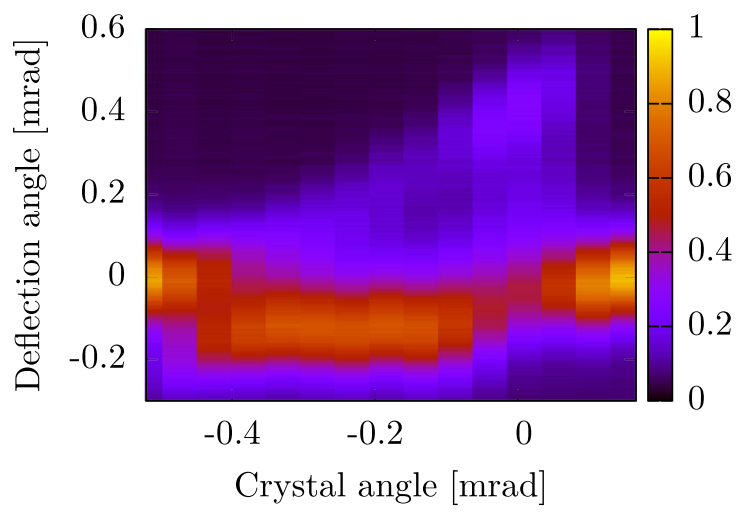

FIG. 4. The probability density relative to the maximum probability density of the deflected particles as a function of the crystal angle for the case of $3.35 \mathrm{GeV}$ electrons.

occurs when the particle is not aimed directly into the acceptance of the channel, but at an angle less than the total bending of the crystal. A rudimentary model of the volume capture efficiency can be obtained in the following manner. An incoming particle is close to the barrier over a distance on the order of $\theta_{c} R$ and since multiple scattering will randomly increase or decrease the transverse mechanical energy the probability per unit length per particle to become captured is also $\frac{1}{L_{d}}$ and therefore the volume capture efficiency should scale as $\frac{\theta_{c} R}{L_{d}}$ which gives an energy dependence of $E^{-\frac{3}{2}}$, assuming Eq. (7) to be correct $[16,22]$. Particles not captured in this manner will remain unbound, but will be deflected with respect to the incoming beam in a direction opposite to the captured particles. This effect is known as volume reflection (VR) $[23,24]$. In Eq. (5) there is an additional linear term in the potential as compared to the case of a straight crystal which means a volume reflected particle initially starts with transverse mechanical energy far above the potential barrier until reaching the turning point at which the crystal potential causes an additional deflection of at most the critical angle. Therefore one expects the angle of volume reflection to be similar to the critical angle i.e. with an energy dependence of $E^{-\frac{1}{2}}$. Previous experiments with positive particles have found an empirical relation with an additional factor of $\log \left(\frac{R}{R_{c}}\right)$ [22].

\section{Surface transmission}

For a beam with no angular divergence, the surface transmission can be determined analytically. The surface transmission is defined as the fraction of particles that become transversely bound upon entry into the crystal to the total number of incoming particles. Over a single channel the distribution of incoming particles is uniform, therefore the surface transmission is the ratio of the length over which the potential seen in Figure 1 is negative, compared to the length over which the force is periodic 


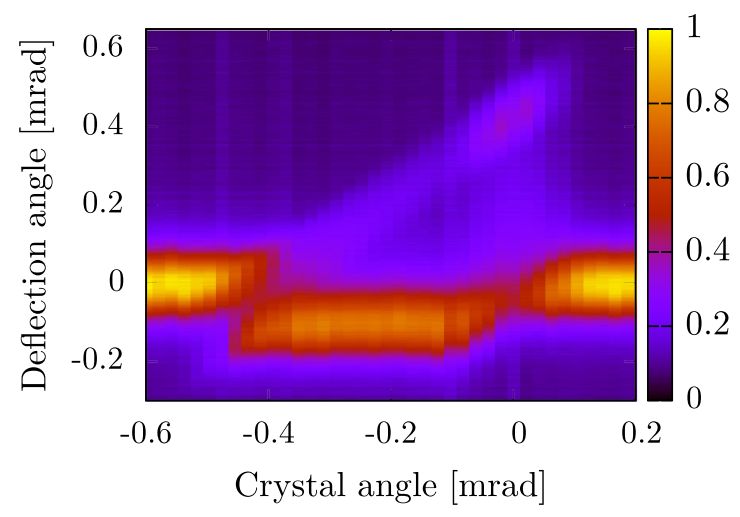

FIG. 5. The probability density relative to the maximum probability density of the deflected particles as a function of the crystal angle for the case of $4.2 \mathrm{GeV}$ electrons.

which is $2 d_{p}$. By approximating the potential in Figure 1 by parabolas around the point $-d_{p}$ and $d_{p}$ one can determine the surface transmission. We write

$$
\begin{aligned}
U(x)= & -\frac{U_{0}}{d_{p}^{2}}\left(x+d_{p}\right)^{2} \operatorname{rect}\left(\frac{x+d_{p}}{2 d_{p}}\right) \\
& -\frac{U_{0}}{d_{p}^{2}}\left(x-d_{p}\right)^{2} \operatorname{rect}\left(\frac{x-d_{p}}{2 d_{p}}\right)+\frac{E x}{R},
\end{aligned}
$$

where $\operatorname{rect}(x)$ is the rectangular function which is 1 of $|x|<\frac{1}{2}$ and 0 otherwise. The leftmost limit of acceptance is determined by the leftmost point $a$ where $U^{\prime}(a)=0$ and the rightmost limit $b$ by the condition that $U(b)=U(a)$. The acceptance factor is then $\frac{b-a}{2 d_{p}}=1-\sqrt{\frac{R_{c}}{R}}$. Including the constant fraction that dechannel due to thermal vibration of the nuclei as seen in [16] we obtain

$$
A=\left(1-\frac{2 u}{d_{p}}\right)\left(1-\sqrt{\frac{R_{c}}{R}}\right)
$$

where $u=0.062 \AA$ is the thermal vibrational amplitude for $\mathrm{Si}$ at room temperature. In Figure 2 we show this formula compared with the exact result obtained numerically using the Doyle-Turner potential.

TABLE II. A table of the theoretical values for the energies of the experiment.

\begin{tabular}{lrrccc}
\hline \hline$E[\mathrm{GeV}]$ & 3.35 & 4.2 & 6.3 & 10.5 & 14.0 \\
\hline$L_{d, b}[\mu \mathrm{m}]$ & 77 & 92 & 124 & 157 & 155 \\
$A[\%]$ & 68 & 65 & 59 & 49 & 42 \\
$\theta_{c}$ & 122 & 109 & 89.1 & 69.0 & 59.8 \\
$R_{c}[\mathrm{~cm}]$ & 0.79 & 0.99 & 1.48 & 2.47 & 3.29 \\
\hline \hline
\end{tabular}

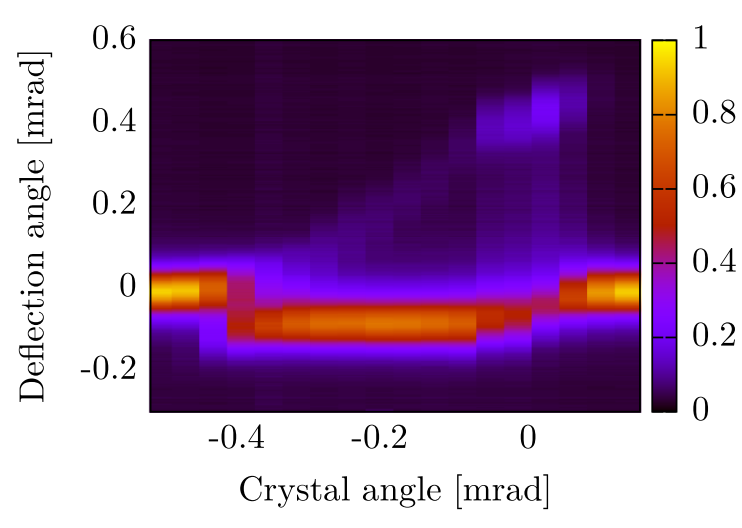

FIG. 6. The probability density relative to the maximum probability density of the deflected particles as a function of the crystal angle for the case of $6.3 \mathrm{GeV}$ electrons.

In Table II we have calculated the values of some of the important parameters for the energies of the experiment.

\section{Simulations}

We will compare the experimental measurements with the simulation code DYNECHARM++ [25]. The code allows the tracking of a relativistic charged particle inside a crystalline medium via the numerical integration of the classical equations of motion. The continuum potential approximation proposed by Lindhard is used [21]. The electrical characteristics of the crystal within this approximation are computed by means of the ECHARM code [26]. Since the multiple and single scatterings may cause the particle to dechannel/rechannel, such incoherent scattering has been included into the simulation. Since the encountered number of nuclei and electrons depends on the particle trajectory [21], in the simulation the normalized distribution of scattering angles [27] scales with the ratio between the density of matter encountered by a particle and the average density of the material. Moreover, the experimental measurements were compared with the results of the Geant4 Monte Carlo toolkit [28], which allows the simulation of the coherent effects of charged particles in crystals [29].

\section{EXPERIMENTAL SETUP AND PROCEDURE}

The silicon ( $\mathrm{Si}$ ) crystal used in this experiment was fabricated [30] at the Sensors and Semiconductor Laboratory at the University of Ferrara with crystallographic orientation chosen to produce quasimosaic bending

TABLE III. A table of the fitting parameters used to describe the distribution in the "amorphous" orientation.

\begin{tabular}{llllll}
\hline \hline$E[\mathrm{GeV}]$ & 3.35 & 4.2 & 6.3 & 10.5 & 14.0 \\
\hline$A$ & 0.571 & 0.776 & 0.872 & 0.696 & 0.77 \\
$r$ & 1.52 & 2.19 & 2.04 & 2.27 & 3.10 \\
\hline \hline
\end{tabular}


of the (111) plane [31]. Its thickness was measured interferometrically to be $60 \pm 1 \mu \mathrm{m}$. The (111) plane has a bending radius of $0.15 \mathrm{~m}$ giving a total bending angle of the crystal of $\theta_{b}=402 \pm 9 \mu \mathrm{rad}$ in the horizontal direction. The crystal was mounted in a scattering chamber in the End Station Test Beam A (ESTB A) at SLAC, see Figure 3. A rotational stage allows rotation of the crystal with step sizes of approximately $5 \mu \mathrm{rad}$. A translational stage moves the crystal to its optimal position. The rotation angle of the crystal is determined by reflecting a laser beam off a flat mirror mounted on the side of the crystal holder. The reflected laser beam hits a screen approximately $1 \mathrm{~m}$ from the mirror. When the crystal is rotated, the laser beam on the screen provides a read-out of the rotation angle of the crystal with a resolution better than $5 \mu \mathrm{rad}$. A Cerium doped Yttrium Aluminum Garnet (YAG for short) screen of $500 \mu \mathrm{m}$ thickness with a CCD camera $13 \mathrm{~m}$ down stream of the crystal provides the means of data acquisition in this experiment. Saturation of the YAG screen is negligible at the bunch charge $10^{8}$ particles per bunch that was provided for this experiment [32]. The beam divergence was measured to be less than $10 \mu \mathrm{rad}$ rms by the wire scanners. The spot-size was less than $150 \mu \mathrm{m}$ and the momentum spread reduced to about $0.15 \%$.

\section{DATA ANALYSIS AND RESULTS}

The experimental measurements were performed by rotating the crystal in small angular steps and recording an image of the circular YAG screen. The camera was mounted at an angle with respect to the screen which distorted the image. An ellipse was fitted along the edge of the screen, for this was known to be circular. This allows one to revert this image distortion due to the positioning of the camera. A region of the screen is chosen such that the edge of the screen is avoided. The crystal deflects particles in the horizontal plane and we sum the intensity in the vertical plane and normalize the probability distribution as seen in e.g. Figure 8 . For each crystal angle several images

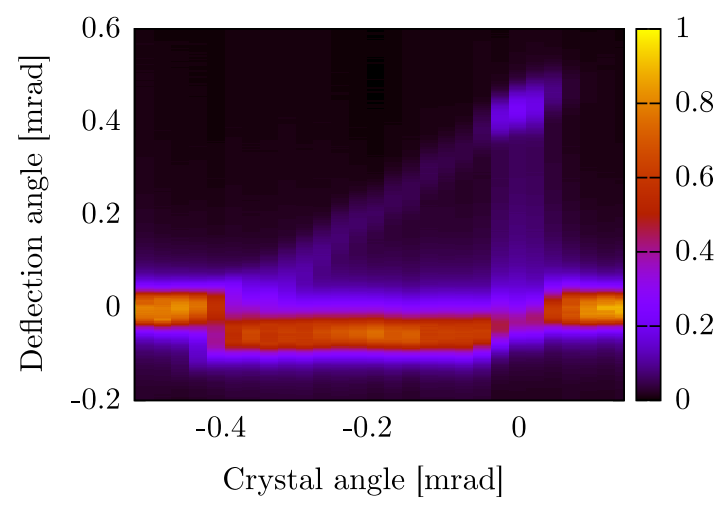

FIG. 7. The probability density relative to the maximum probability density of the deflected particles as a function of the crystal angle for the case of $10.5 \mathrm{GeV}$ electrons. were taken. Images with low or high (camera saturated) light intensities were ignored. Plotting the distribution along the $\mathrm{y}$-axis with the crystal angle along the $\mathrm{x}$-axis one obtains the so called "triangle plots," (Figures 2, 4, 5, 6, and 7 represent the raw data). A crystal angle of 0 was chosen to be the orientation of closest direct entry of the beam into the channel as could be experimentally realized. This orientation, along with the halfway of the full bending of $\theta_{b}=402 \mu \mathrm{rad}$, (roughly $\frac{\theta_{b}}{2}$ ) termed the volume reflection orientation, will be investigated in detail. In the triangle plots of Figure 4 to Figure 7 some features should be noted. When the beam has a tangent along the curved crystal plane, volume reflection takes place giving rise to the downshifted horizontal island in these figures. The skewed line shows the particles not reflected at the tangent between the bent plane and beam, but captured and following the curvature until the end of the crystal. The small island at the end of the skewed line arises due to particles entering directly into the crystal channel acceptance at the crystal face, and which travel along the whole curvature of the crystal plane. The vertical line connecting this island and the lower horizontal island are the particles that were captured at the crystal face, but then dechannel along the way.

In Figure 8 and Figure 9 the probability density of the deflected particles is plotted for the two different cases described above. In Figure 8, some general tendencies can be identified. The width of the large leftmost peak, becomes narrower as does the channeled peak due to the decreasing critical angle. In Figure 9 the large leftmost peak due to volume reflection moves closer to the undeflected position and the width decreases as energy increases. To extract quantitative information from these distributions we consider a fitting procedure consisting of two Gaussian probability distributions for the two peaks and a function for the dechanneled particles in between to be specified. For $i=1,2$ we have two Gaussian distributions

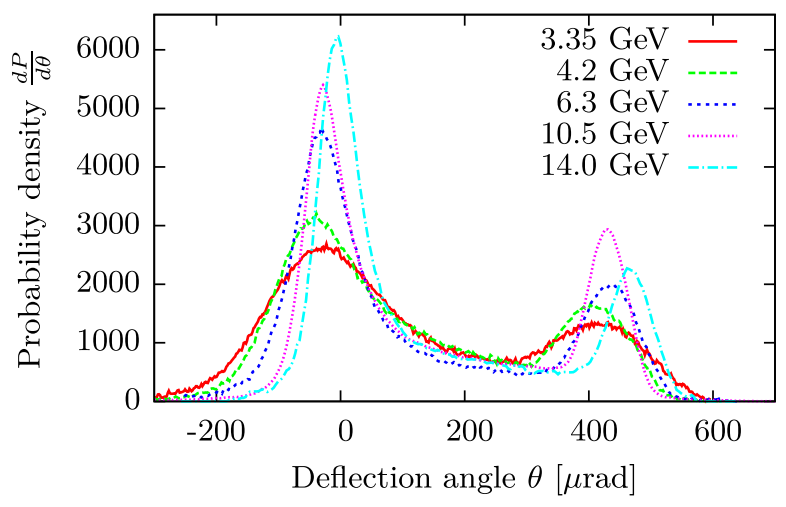

FIG. 8. The probability density of the deflected particles when the crystal is in the channeling orientation for the different energies in the experiment. 


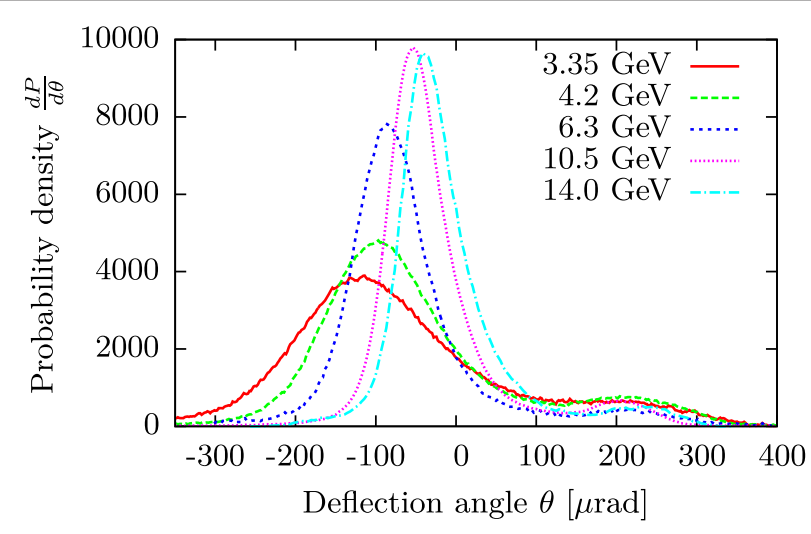

FIG. 9. The probability density of the deflected particles when the crystal is at half of the full bending angle. The large leftmost peaks are the volume reflected portions and the small rightmost peaks are the volume captured particles.

$$
\frac{d P}{d \theta}(\theta)=\frac{P_{i}}{\sigma_{i} \sqrt{2 \pi}} e^{-\frac{\left(\theta-\mu_{i}\right)^{2}}{2 \sigma_{i}^{2}}},
$$

where $P_{i}$ is the fraction of particles in this peak, $\sigma_{i}$ the standard deviation of the distribution and $\mu_{i}$ the center. The undeflected/volume reflected particles are denoted by $i=1$ and either the channeled or volume captured particles are denoted by $i=2$. The probability to dechannel per angle in the exponential decay model of Eq. (8) is $\frac{d P}{d \theta}=e^{-\frac{\theta}{\theta_{d}}} / \theta_{d}$. Therefore the probability distribution of the dechanneled particles becomes

$$
\frac{d P_{d}}{d \theta}(\theta)=\int_{\mu_{1}}^{\mu_{2}} \frac{1-P_{1}}{\sigma_{2} \sqrt{2 \pi}} e^{-\frac{\left(\theta-\theta^{\prime}\right)^{2}}{2 \sigma_{2}^{2}}} \frac{1}{\theta_{d}} e^{-\frac{\theta^{\prime}}{\theta_{d}}} d \theta^{\prime},
$$

where $\theta_{d}=\frac{L_{d}}{L} \theta_{b}$. This formula can be understood as follows: The probability to find a particle at an angle $\theta$ is the sum over the possible ways this can happen. A fraction of the particles dechanneling at angle $\theta^{\prime}$ can end up at the angle $\theta$ due to the distribution of particles in the channel. The above can also be rewritten as

$$
\begin{aligned}
\frac{d P_{d}}{d \theta}(\theta)= & \frac{1-P_{1}}{2 \theta_{d}} e^{\frac{\sigma_{2}^{2}}{2 \theta_{d}^{2}}+\frac{\mu_{1}}{\theta_{d}}-\frac{\theta}{\theta_{d}}} \\
& \times\left(\operatorname{erf}\left(\frac{\mu_{2}-\Delta \theta}{\sqrt{2} \sigma_{2}}\right)-\operatorname{erf}\left(\frac{\mu_{1}-\Delta \theta}{\sqrt{2} \sigma_{2}}\right)\right),
\end{aligned}
$$

where $\Delta \theta=\theta-\frac{\sigma_{2}^{2}}{\theta_{d}}$. The exponential dechanneling model means that $P_{2}$ is given by $P_{2}=\left(1-P_{1}\right) e^{-\frac{L}{L_{d}}}$. This model therefore contains six free parameters: $P_{1}, \sigma_{1}$, $\mu_{1}, \sigma_{2}, \mu_{2}, L_{d}$. It turns out that the non-Gaussian tail of the undeflected peak has a large influence on the fit between the two peak functions which is important when trying to determine the dechanneling length. A

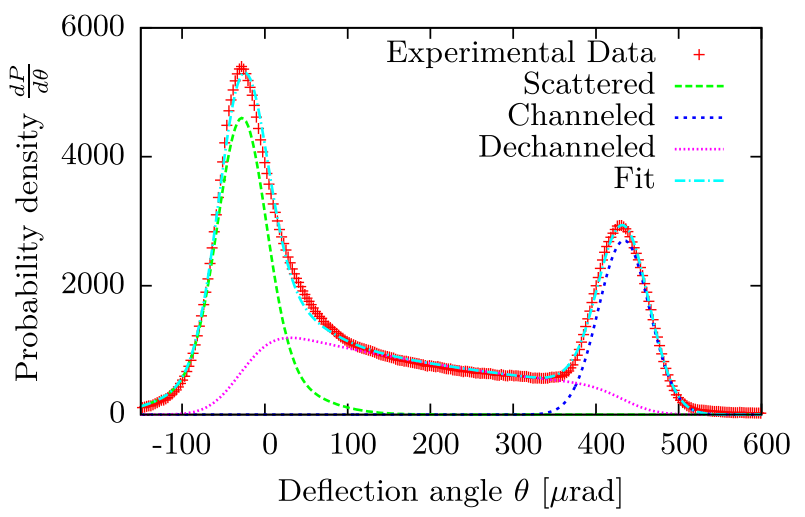

FIG. 10. A fit to the $10.5 \mathrm{GeV}$ experimental data in the channeling orientation.

sum of two Gaussians fits the peak in the "amorphous" orientation very well which we write in the form

$\frac{d P}{d \theta}(\theta)=P_{1}\left(\frac{A}{\sigma_{1} \sqrt{2 \pi}} e^{-\frac{\left(\theta-\mu_{1}\right)^{2}}{2 \sigma_{1}^{2}}}+\frac{1-A}{r \sigma_{1} \sqrt{2 \pi}} e^{-\frac{\left(\theta-\mu_{1}\right)^{2}}{2 r_{1}^{2}}}\right)$,

where the standard deviation of the second Gaussian is $r \sigma_{1}$. The values of $A$ and $r$ were then found by performing a fit in the amorphous orientation and we obtain the values found in Table 3 .

In Figure 10 and Figure 11 the data in the "channeling" orientation for the lowest and a high energy are fitted according to the model described here. The results are shown in Table IV.

Performing the same type of fit to the results of the simulations shown in Figure 14 one obtains the results shown in Table V.

The errors given here are only statistical. The simulations were performed with beam angular divergences of $30 \mu \mathrm{rad}$, $27.5 \mu \mathrm{rad}, 21.5 \mu \mathrm{rad}, 10 \mu \mathrm{rad}$, and $10 \mu \mathrm{rad}$ for the energies $3.35 \mathrm{GeV}, 4.2 \mathrm{GeV}, 6.3 \mathrm{GeV}, 10.5 \mathrm{GeV}$, and $14.0 \mathrm{GeV}$, respectively.

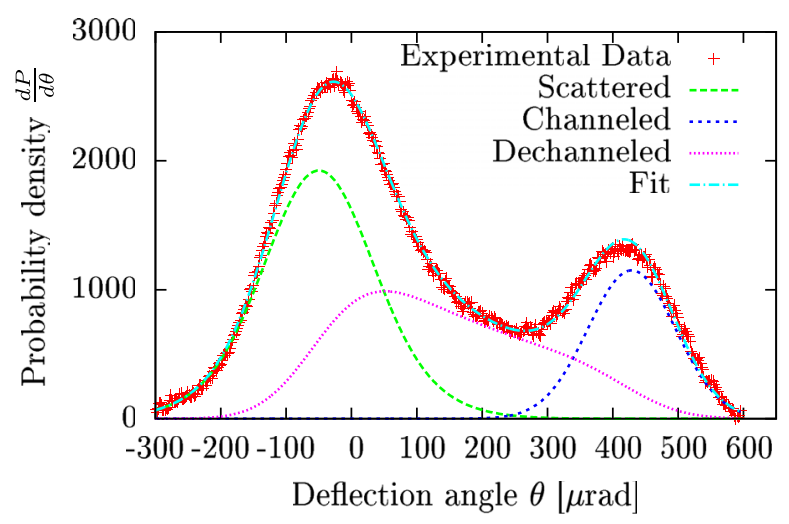

FIG. 11. A fit to the $3.5 \mathrm{GeV}$ experimental data in the channeling orientation. 
TABLE IV. A table of the fitting parameters for the crystal in "channeling" orientation using the described model.

\begin{tabular}{|c|c|c|c|c|c|}
\hline$E[\mathrm{GeV}]$ & 3.35 & 4.2 & 6.3 & 10.5 & 14.0 \\
\hline$L_{d}[\mu \mathrm{m}]$ & $55.4 \pm 1.8$ & $45.2 \pm 1.2$ & $65.3 \pm 1.9$ & $57.5 \pm 1.2$ & $55.8 \pm 1.2$ \\
\hline$P_{1}[\%]$ & $43 \pm 1.3$ & $33 \pm 1.5$ & $50 \pm 0.8$ & $41 \pm 0.7$ & $49 \pm 0.7$ \\
\hline$\mu_{1}[\mu \mathrm{rad}]$ & $-49.9 \pm 2.3$ & $-62.4 \pm 2.3$ & $-33.8 \pm 1.0$ & $-28.4 \pm 0.8$ & $0.29 \pm 0.5$ \\
\hline$\mu_{2}[\mu \mathrm{rad}]$ & $428.8 \pm 1.45$ & $419.8 \pm 1.5$ & $439.2 \pm 1.2$ & $433.9 \pm 0.8$ & $470.3 \pm 0.9$ \\
\hline$\sigma_{1}[\mu \mathrm{rad}]$ & $76.1 \pm 0.92$ & $53.9 \pm 1.1$ & $49.2 \pm 0.8$ & $29.0 \pm 0.5$ & $29.0 \pm 0.5$ \\
\hline$\sigma_{2}[\mu \mathrm{rad}]$ & $67.0 \pm 1.1$ & $48.5 \pm 1.0$ & $42.6 \pm 0.9$ & $30.9 \pm 0.6$ & $34.1 \pm 0.7$ \\
\hline
\end{tabular}

TABLE V. A table of the fitting parameters for the crystal DYNECHARM simulation in channeling orientation using the described model.

\begin{tabular}{|c|c|c|c|c|c|}
\hline$E[\mathrm{GeV}]$ & 3.35 & 4.2 & 6.3 & 10.5 & 14.0 \\
\hline$L_{d}[\mu \mathrm{m}]$ & $39.4 \pm 0.3$ & $47.4 \pm 0.5$ & $54.3 \pm 0.5$ & $57.1 \pm 0.6$ & $48.1 \pm 0.8$ \\
\hline$P_{1}[\%]$ & $11 \pm 0.9$ & $27 \pm 0.7$ & $34 \pm 0.4$ & $38 \pm 0.3$ & $40.1 \pm 0.6$ \\
\hline$\mu_{1}[\mu \mathrm{rad}]$ & $-97.6 \pm 2.1$ & $-57.0 \pm 2.3$ & $-37.5 \pm 0.5$ & $-25.5 \pm 0.3$ & $-20.6 \pm 0.4$ \\
\hline$\mu_{2}[\mu \mathrm{rad}]$ & $441.4 \pm 0.5$ & $439.2 \pm 0.5$ & $438.7 \pm 0.3$ & $437.6 \pm 0.3$ & $437.4 \pm 0.5$ \\
\hline$\sigma_{1}[\mu \mathrm{rad}]$ & $78.1 \pm 3.6$ & $56.0 \pm 0.9$ & $39.4 \pm 0.3$ & $24.8 \pm 0.2$ & $16.8 \pm 0.3$ \\
\hline$\sigma_{2}[\mu \mathrm{rad}]$ & $50.4 \pm 0.7$ & $42.8 \pm 0.6$ & $31.9 \pm 0.2$ & $21.5 \pm 0.2$ & $17.4 \pm 0.3$ \\
\hline
\end{tabular}

TABLE VI. A table of the fitting parameters for the crystal in "volume capture" orientation using the described model.

\begin{tabular}{|c|c|c|c|c|c|}
\hline$E[\mathrm{GeV}]$ & 3.35 & 4.2 & 6.3 & 10.5 & 14.0 \\
\hline$L_{d}[\mu \mathrm{m}]$ & $35.5 \pm 3.9$ & $45.6 \pm 3.1$ & $36.4 \pm 3.8$ & $40.9 \pm 3.4$ & $25.6 \pm 3.7$ \\
\hline$P_{1}[\%]$ & $67 \pm 3.8$ & $73 \pm 1.3$ & $84 \pm 1.1$ & $84 \pm 0.9$ & $80.7 \pm 2.3$ \\
\hline$\mu_{1}[\mu \mathrm{rad}]$ & $-128.8 \pm 4.1$ & $-100.7 \pm 1.2$ & $-84.0 \pm 0.9$ & $-50.3 \pm 0.6$ & $-35.6 \pm 1.4$ \\
\hline$\mu_{2}[\mu \mathrm{rad}]$ & $242.1 \pm 5.3$ & $239.9 \pm 3.6$ & $243.3 \pm 8.1$ & $219.8 \pm 3.7$ & $258.0 \pm 9.5$ \\
\hline$\sigma_{1}[\mu \mathrm{rad}]$ & $73.5 \pm 1.2$ & $58.1 \pm 0.7$ & $42.8 \pm 0.7$ & $28.5 \pm 0.4$ & $28.2 \pm 0.9$ \\
\hline$\sigma_{2}[\mu \mathrm{rad}]$ & $57.9 \pm 3.0$ & $55.8 \pm 2.3$ & $55.0 \pm 5.6$ & $32.9 \pm 2.6$ & $27.0 \pm 6.1$ \\
\hline
\end{tabular}

Similarly in Figure 12 and Figure 13, the same fit is applied to the case where the crystal is roughly at the angle of $\frac{\theta_{b}}{2}$ and the results are shown in Table VI.

It is important to note that the model presented here is different than the one used for the analysis of the 3.35 and $6.3 \mathrm{GeV}$ data seen in [14]. Therefore different numbers for e.g. the dechanneling length is obtained. In particular the approach using a double Gaussian for the amorphous/VR peak is a difference and that we in the model used in this paper have one less fitting parameter. This model is thus more constrained. In [14] it was noted that the dechanneling length could be determined in two ways. One based on the shape of the distribution (e.g. Figure 11) between the two peaks, and another based on the relative sizes of the two peaks. In [14] this gave rise to two different dechanneling lengths. The model used here is constrained such that this is not a possibility. Using one less fitting parameter it is expected that the fit could be worse. However, generally the fits are good, but worse for higher energy than at low energies ( 10.5 and $14 \mathrm{GeV}$ are about equally good). Only in the case of the VR orientation at $14 \mathrm{GeV}$ is the fit worse than the example shown by Figure 12 but this also to a considerable degree.

\section{A. Multiple scattering of channeled particles parallel to the plane}

In Figure 15 we have plotted the r.m.s. standard deviation, $\sigma_{\|}$, of the Gaussian function fitted to the distribution of channeled particles in the direction parallel to the plane i.e. in the "free" direction in the continuum potential approximation. It is therefore expected that multiple scattering determines the width in this case. The multiple scattering width scales as $1 / E$ and performing a fit we obtain the best fit to be given by

$$
\sigma_{\mathrm{MS}}=\frac{520 \mu \mathrm{rad} \times \mathrm{GeV}}{E}
$$




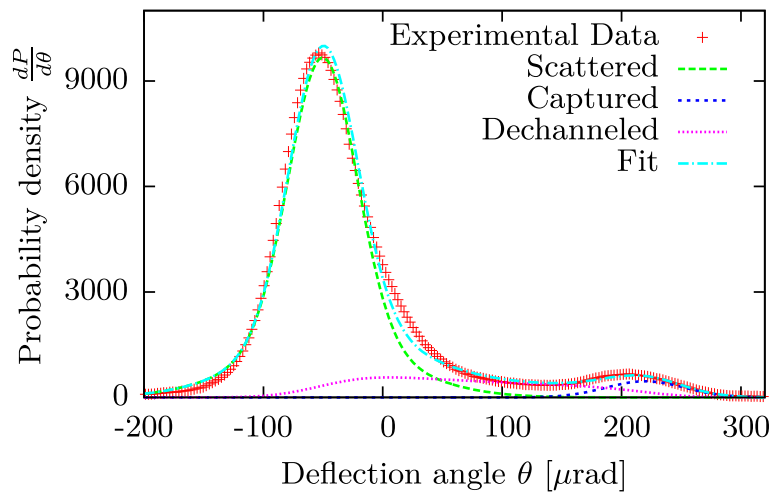

FIG. 12. A fit to the $10.5 \mathrm{GeV}$ experimental data at half the crystal bending angle.

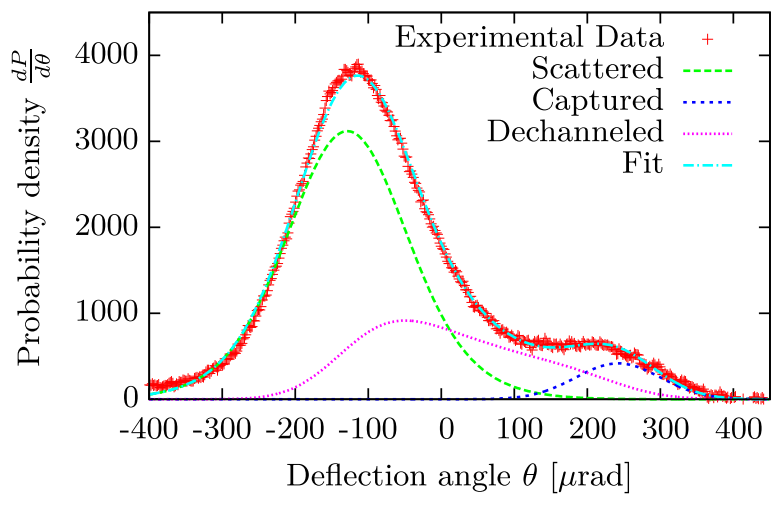

FIG. 13. A fit to the $3.5 \mathrm{GeV}$ experimental data at half the crystal bending angle.

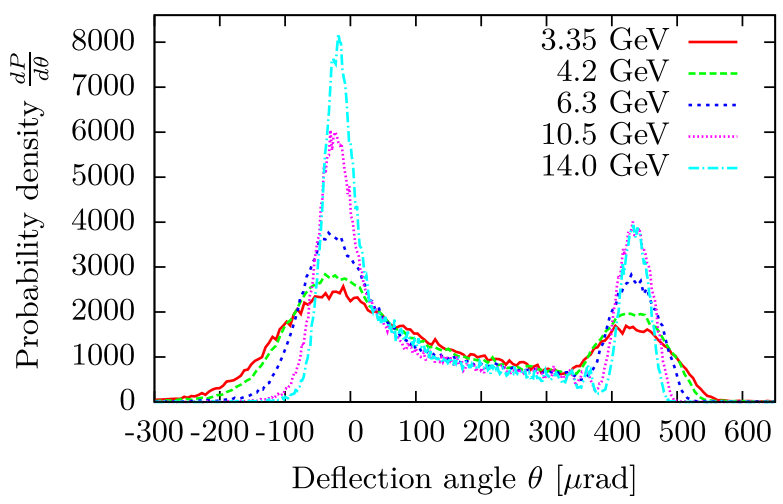

FIG. 14. The probability density of the deflected particles obtained from the DYNECHARM simulation when the crystal is in the channeling orientation for the different energies in the experiment.

The usual formula [27] for multiple scattering would predict the factor to be $302 \mu \mathrm{rad} \times \mathrm{GeV}$, thus an increased scattering is observed. This is likely due to negative particles crossing the plane during channeling oscillations and thus more often encounter hard scattering with the nuclei $[18,29,33]$. We are not aware of a derived theoretical formula

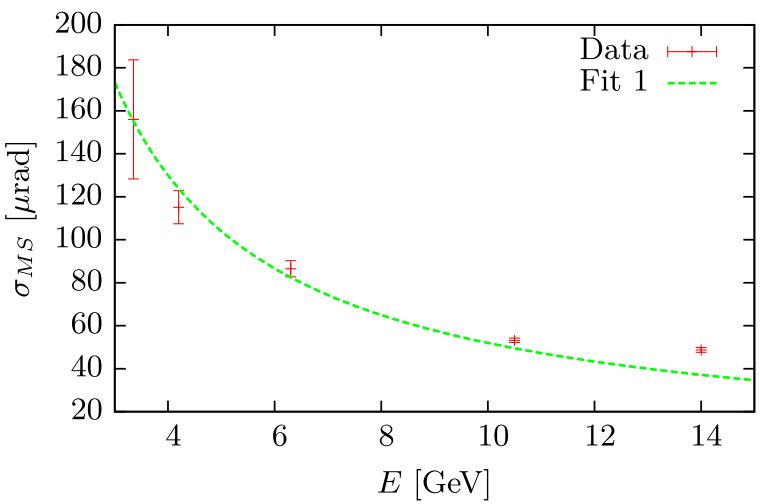

FIG. 15. The width of the channeling peak in the direction parallel to the plane is fitted to obtain Eq. (16).

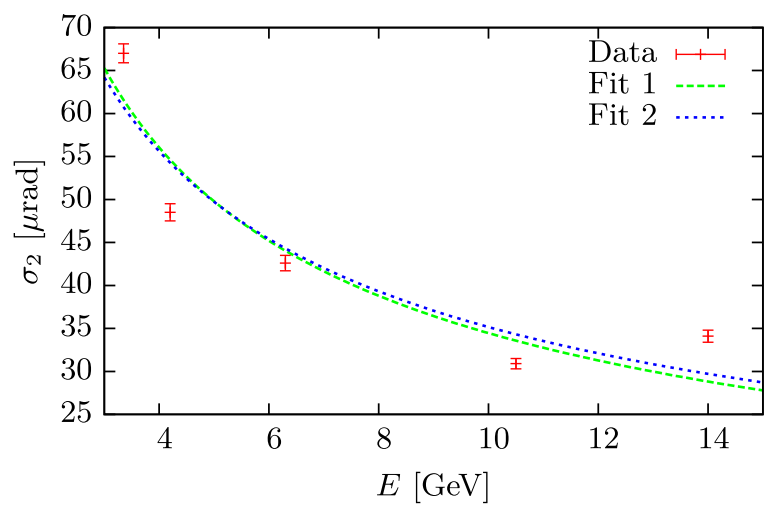

FIG. 16. The width of the channeling peak in the direction orthogonal to the plane is fitted with a power function (Fit 1), see Eq. (17) and to a function of the type $a / \sqrt{E}$ (Fit 2), see Eq. (18).

which predicts the size of this effect and it seems difficult to do so since channeling is usually described by the continuum potential which neglects incoherent scattering. This effect is an interplay between the incoherent scattering and the channeling motion which are usually described separately. Simulation of the effect should, however, be possible.

\section{B. Angular distribution of channeled particle orthogonal to the plane}

In Figure 16 we have plotted the r.m.s. standard deviations $\sigma_{2}$ from Table IV and a fit to a power function that yields

$$
\sigma_{2}=117 \mu \mathrm{rad} \times E[\mathrm{GeV}]^{-0.53} .
$$

Since the critical angle scales as $1 / \sqrt{E}$ we have also performed such a fit to obtain

$$
\sigma_{2}=111 \mu \mathrm{rad} \times E[\mathrm{GeV}]^{-0.5} .
$$

The scatter of the points around these curves indicates a systematic error which is likely due to the limited resolution of the rotational stage, i.e. it is not guaranteed that the 
crystal is at exactly the same angle to the beam for each energy.

\section{Deflection angle of volume reflected particles}

In Figure 17 we have plotted the deflection angle in volume capture orientation $\left(-\mu_{1}\right.$ from Table VI) versus the beam energy and fit the data to two different functions. Fitting the data to a power function we obtain

$$
\left|\mu_{1}\right|=338 \mu \mathrm{rad} \times E[\mathrm{GeV}]^{-0.81} .
$$

Again, we would expect there to be a $1 / \sqrt{E}$ scaling. With such a fit we obtain

$$
\left|\mu_{1}\right|=207 \mu \mathrm{rad} \times E[\mathrm{GeV}]^{-0.5} .
$$

\section{Efficiency of volume capture}

In the case of "volume reflection orientation," $P_{1}$ gives the fraction of reflected particles and the rest will have been captured, such that $1-P_{1}$ is the fraction of particles captured. From Sec. II, we expect the volume reflection to scale with energy as $E^{-3 / 2}$ [20]. We have therefore performed such a fit along with a fit to a power function, see Figure. 18, and obtained

$$
\varepsilon_{\mathrm{VR}}=2.3 \times E[\mathrm{GeV}]^{-1.5},
$$

and

$$
\varepsilon_{\mathrm{VR}}=0.57 \times E[\mathrm{GeV}]^{-0.52} .
$$

It is seen that the fitted power function is close to a $1 / \sqrt{E}$ scaling. If one assumes an energy independent dechanneling length $L_{D}$ as is indicated experimentally, one would also expect the volume capture efficiency to instead scale as $1 / \sqrt{E}$. Although this observation may be surprising, the

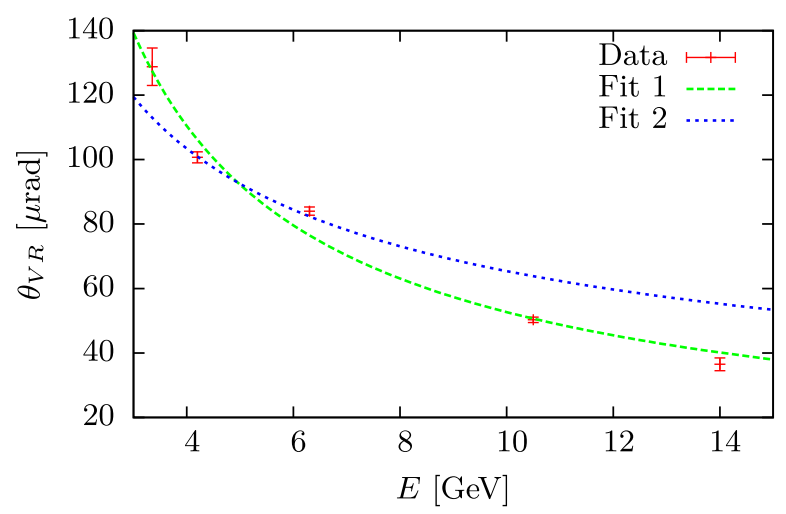

FIG. 17. The angle of volume reflection is fitted with a power function (Fit 1) and to a function of the type $a / \sqrt{E}$ (Fit 2).

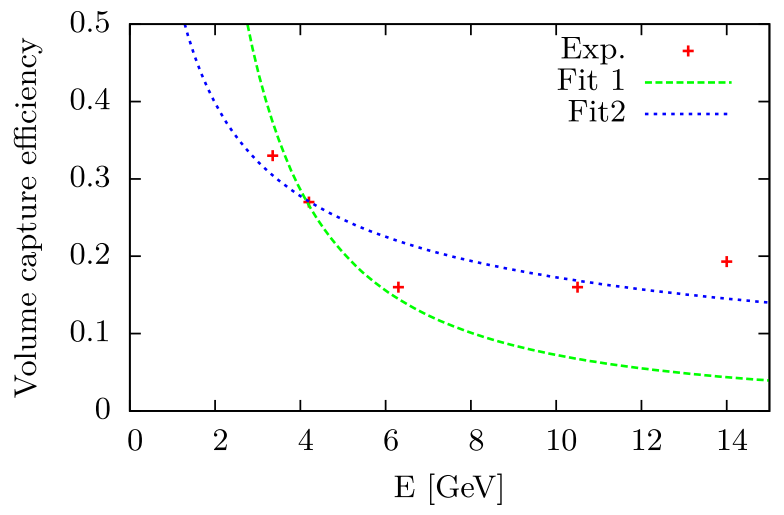

FIG. 18. The volume capture efficiency as given by $1-P_{1}$ from Table VI. "Fit 1 " is a fit to a function of the type $a E^{-3 / 2}$ and "Fit 2" is a fit to a power function $\left(a E^{b}\right)$.

data set thus consistently shows a dechanneling length that is largely independent on energy.

\section{E. Discussion of the dechanneling length and surface transmission}

In Table V and Figure 19, supported by the fit in Figure 18, as discussed, it is evident that the dechanneling lengths obtained from the simulation has a dependence on energy which is not directly proportional as the simple formula of Eq. (7) prescribes. The formula which has been corrected for the crystal bending Eq. (9) however, has a qualitative agreement. Based on this we performed a fit where we allowed for a constant scaling front factor, and a variable scale factor on $R_{c}$, determined by the fit. This yields

$$
L_{D}=15.3 \frac{\mu \mathrm{m}}{\mathrm{GeV}} \cdot E\left(1-1.76 \frac{2 R_{c}}{R}\right) .
$$

In Eq. (9) the front factor is $20.3 \frac{\mu \mathrm{m}}{\mathrm{GeV}}$. Keeping in mind the simple derivation leading to Eq. (7), an accuracy to

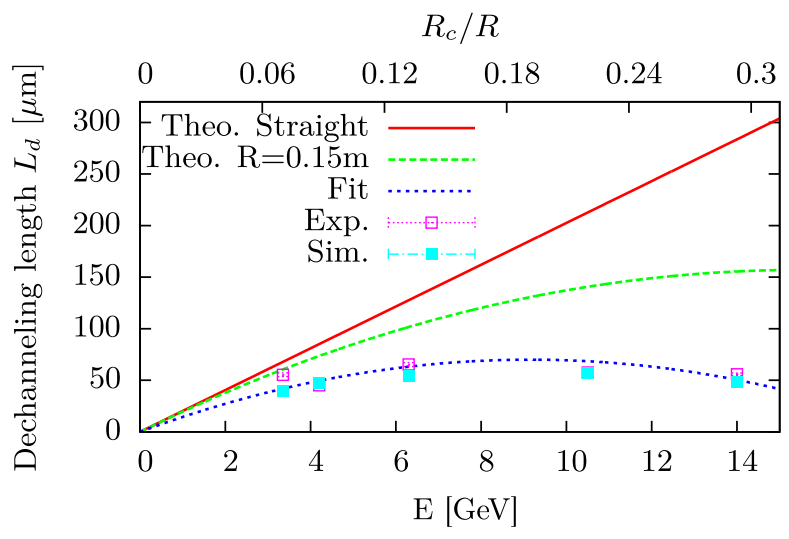

FIG. 19. A plot of the "simple" dechanneling formulas of Eqs. (7), (9), (23) and of the experimental and simulated values. 


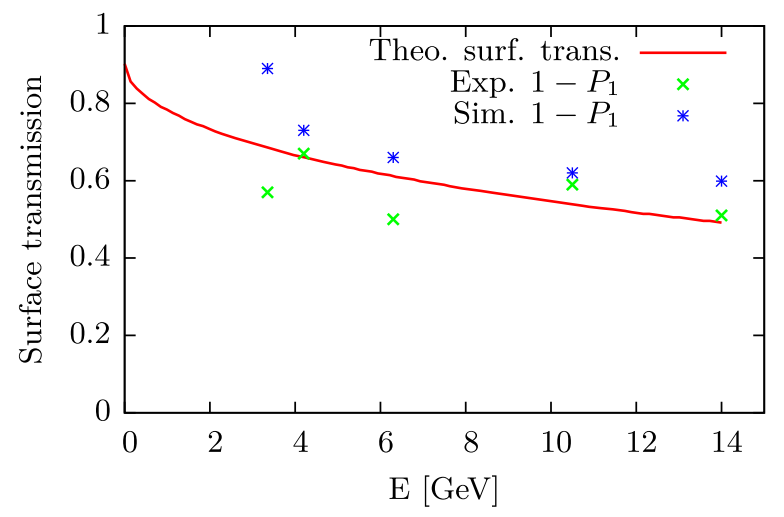

FIG. 20. The experimental and simulated values of the surface transmission given by $1-P_{1}$ is compared with the model of Eq. (11).

higher than a factor of 2 cannot be expected, but should give the correct scaling and order of magnitude. Eq. (23) should thus be usable as a predictive formula for other energies and bending radii of the Si (111) plane.

The measured and calculated [from Eq. (11)] values of the surface transmission are in good agreement and are plotted in Figure 20. In this case the fluctuation of the data points around this curve we also attribute to the uncertainty on the beam entry angle.

\section{CONCLUSION}

We have presented a detailed experimental study of the deflection of electron beams of $\mathrm{GeV}$ energies using bent crystals. Furthermore, we have shown that a fitting procedure based on (1) a simple exponential decay model of channeled particles and (2) that these particles are distributed according to a Gaussian distribution within the channel, fit the data with good agreement. Based on the parameters of the fitting functions we have extracted important parameters describing the channeling process, such as the dechanneling length, the angle of volume reflection, the surface transmission and the widths of the distribution of channeled particles parallel and orthogonal to the plane. The scattering parallel to the plane fits well with the usual functional dependence of multiple scattering on energy but is larger than amorphous/random by a factor of 1.7. For the distribution of channeled particles, the mean of the angle of volume reflection and the efficiency of volume capture, were fit to power functions with a free exponent and to a exponent fixed by that predicted by "simple" theory. In these cases, a significant deviation is seen in the exponent which indicates that the simple theory may be too simple to describe the results of this experiment. The simple formula for surface transmission, however, works out well. The dechanneling lengths observed in these experiments are significantly shorter than predicted by the simple theory and the dechanneling length in "VR orientation" is seen to be consistently smaller than in "channeling orientation" indicating preferential population of high-lying transverse energy states in VR mode. However, in the DYNECHARM simulation, good agreement is seen in the angular distributions of the exiting particles and the obtained dechanneling lengths. It would be interesting if a similar experiment is conducted with positrons instead.

This work was partially supported by the U.S. DOE under Contract No. DE- AC02-76SF00515, by the U.S. National Science Foundation (Grant No. PHY-1068662), by the Danish Council for Independent Research-Natural Sciences FNU, and the Italian Istituto Nazionale di Fisica Nucleare (INFN) through the CHANEL experiment. We wish to acknowledge also Mr. Persiani Andrea and Mr. Manfredi Claudio of Perman (Loiano, Italy) for their support with crystal holders manufacturing. Gilles Frequet from Fogale Nanotech for precise measurement of crystal thickness by means of a T-MAP IR interferometer.

[1] W. Scandale et al., Deflection of $400 \mathrm{GeV} / c$ proton beam with bent silicon crystals at the CERN Super Proton Synchrotron, Phys. Rev. ST Accel. Beams 11, 063501 (2008).

[2] R. A. Carrigan et al., Beam extraction studies at $900 \mathrm{GeV}$ using a channeling crystal, Phys. Rev. ST Accel. Beams 5, 043501 (2002).

[3] S. Møller, E. Uggerhøj, H. Atherton, M. Clément, N. Doble, K. Elsener, L. Gatignon, P. Grafström, M. HageAli, and P. Siffert, High efficiency bending of $450 \mathrm{GeV}$ protons using channeling, Phys. Lett. B 256, 91 (1991).

[4] N. Mokhov et al., Charged and Neutral Particles Channeling Phenomena (World Scientific, Singapore, 2012), Chap. 15, p. 172.

[5] N. Mokhov, J. Annala, R. Carrigan, M. Church, A. Drozhdin, T. Johnson, R. Reilly, V. Shiltsev, G. Stancari, D. Still, A. Valishev, X. L. Zhang, and V. Zvoda, Tevatron beam halo collimation system: design, operational experience and new methods, J. Instrum. 6, T08005 (2011).

[6] R. Carrigan, V. Lebedev, N. Mokhov, S. Nagaitsev, V. Shiltsev, G. Stancari, D. Still, and A. Valishev, Accelerator Physics at the Tevatron Collider (Springer, New York, 2014), p. 187.

[7] U. I. Uggerhøj, The interaction of relativistic particles with strong crystalline fields, Rev. Mod. Phys. 77, 1131 (2005).

[8] T. N. Wistisen, K. K. Andersen, S. Yilmaz, R. Mikkelsen, J. L. Hansen, U. I. Uggerhøj, W. Lauth, and H. Backe, Experimental Realization of a New Type of Crystalline Undulator, Phys. Rev. Lett. 112, 254801 (2014).

[9] A. Kostyuk, Recent progress in the theory of the crystalline undulator, Nucl. Instrum. Methods Phys. Res., Sect. B 309, 45 (2013).

[10] U. Mikkelsen and E. Uggerhøj, A crystalline undulator based on graded composition strained layers in a superlattice, Nucl. Instrum. Methods Phys. Res., Sect. B 160, 435 (2000). 
[11] R. O. Avakyan, L. A. Gevorgyan, K. A. Ispiryan, and R. K. Ispiryan, Radiation from particles in crystal undulators with allowance for a polarization of the medium, J. Exp. Theor. Phys. Lett. 68, 467 (1998).

[12] A. V. Korol, A. V. Solov'yov, and W. Greiner, Coherent radiation of an ultrarelativistic charged particle channelled in a periodically bent crystal, J. Phys. G 24, L45 (1998).

[13] S. Bellucci, S. Bini, V. M. Biryukov, Y. A. Chesnokov, S. Dabagov, G. Giannini, V. Guidi, Y. M. Ivanov, V. I. Kotov, V. A. Maisheev, C. Malagù, G. Martinelli, A. A. Petrunin, V. V. Skorobogatov, M. Stefancich, and D. Vincenzi, Experimental Study for the Feasibility of a Crystalline Undulator, Phys. Rev. Lett. 90, 034801 (2003).

[14] U. Wienands, T. W. Markiewicz, J. Nelson, R. J. Noble, J. L. Turner, U. I. Uggerhøj, T. N. Wistisen, E. Bagli, L. Bandiera, G. Germogli, V. Guidi, A. Mazzolari, R. Holtzapple, and M. Miller, Observation of Deflection of a Beam of Multi-GeV Electrons by a Thin Crystal, Phys. Rev. Lett. 114, 074801 (2015).

[15] S.P. Møller, High-energy channeling-applications in beam bending and extraction, Nucl. Instrum. Methods Phys. Res., Sect. A 361, 403 (1995).

[16] V. Biryukov, Y. Chesnokov, and V. Kotov, Crystal Channeling and its Application at High-Energy Accelerators (Springer-Verlag, Berlin, 1997).

[17] V. Baier, V. Katkov, and V. Strakhovenko, Electromagnetic Processes at High Energies in Oriented Single Crystals (World Scientific, Singapore, 1998).

[18] W. Scandale et al., Measurement of the dechanneling length for high-energy negative pions, Phys. Lett. B 719, 70 (2013).

[19] A. Mazzolari, E. Bagli, L. Bandiera, V. Guidi, H. Backe, W. Lauth, V. Tikhomirov, A. Berra, D. Lietti, M. Prest, E. Vallazza, and D. De Salvador, Steering of a Sub-GeV Electron Beam through Planar Channeling Enhanced by Rechanneling, Phys. Rev. Lett. 112, 135503 (2014).

[20] W. Scandale et al., Volume Reflection Dependence of $400 \mathrm{GeV} / c$ Protons on the Bent Crystal Curvature, Phys. Rev. Lett. 101, 234801 (2008).
[21] J. Lindhard, Influence of crystal lattice on motion of energetic charged particles, Danske Vid. Selsk. Mat. Fys. Medd. 34, 14 (1965).

[22] V. Biryukov, The limits of volume reflection in bent crystals, Nucl. Instrum. Methods Phys. Res., Sect. B 267, 2457 (2009).

[23] A. Taratin and S. Vorobiev, "Volume reflection" of highenergy charged particles in quasi-channeling states in bent crystals, Phys. Lett. 119A, 425 (1987).

[24] W. Scandale et al., Observation of multiple volume reflection by different planes in one bent silicon crystal for high-energy negative particles, Europhys. Lett. 93, 56002 (2011).

[25] E. Bagli and V. Guidi, DYNECHARM++: A toolkit to simulate coherent interactions of high-energy charged particles in complex structures, Nucl. Instrum. Methods Phys. Res., Sect. B 309, 124 (2013).

[26] E. Bagli, V. Guidi, and V. A. Maisheev, Calculation of the potential for interaction of particles with complex atomic structures, Phys. Rev. E 81, 026708 (2010).

[27] J. Beringer et al. (Particle Data Group), Review of particle physics, Phys. Rev. D 86, 010001 (2012).

[28] S. Agostinelli et al., Geant4-a simulation toolkit, Nucl. Instrum. Methods Phys. Res., Sect. A 506, 250 (2003).

[29] E. Bagli, M. Asai, D. Brandt, A. Dotti, V. Guidi, and D. H. Wright, A model for the interaction of high-energy particles in straight and bent crystals implemented in Geant4, Eur. Phys. J. C 74, 2996 (2014).

[30] G. Germogli, A. Mazzolari, L. Bandiera, E. Bagli, and V. Guidi, Manufacturing and characterization of bent silicon crystals for studies of coherent interactions with negatively charged particles beams, Nucl. Instrum. Methods Phys. Res., Sect. B 355, 81 (2015).

[31] R. Camattari, V. Guidi, V. Bellucci, and A. Mazzolari, The 'quasi-mosaic' effect in crystals and its applications in modern physics, J. Appl. Crystallogr. 48, 977 (2015).

[32] J. Rosenzweig and L. Serafini, The Physics of High Brightness Beams (World Scientific, Singapore, 2000).

[33] A. Sørensen and E. Uggerhøj, Channeling, radiation and application, Nucl. Sci. Appl. 3, 147 (1989). 